\title{
Interfaces entre o trabalho e a família: Questões conceituais e empíricas
}

Carolina Villa Nova Aguiar. Escola Bahiana de Medicina e Saúde Pública.

Antonio Virgilio Bittencourt Bastos. Instituto de Psicologia da Universidade Federal da Bahia.

\section{Resumo}

O estudo das interfaces entre o trabalho e a família tem sido conduzido a partir de duas perspectivas antagônicas: de um lado, encontra-se a abordagem do conflito trabalho-família, linha tradicional de pesquisa que considera que as demandas advindas do trabalho e da família são em geral incompatíveis; de outro lado, encontra-se a perspectiva que considera que o acúmulo de papéis ocupacionais e familiares podem ser benéficos para os indivíduos em ambas as esferas da vida. O presente artigo teve o objetivo de caracterizar as duas principais perspectivas teóricas que dominam a pesquisa sobre as interfaces trabalho-família, destacando como problemas conceituais ainda presentes em ambas as vertentes se traduzem em problemas de mensuração, especialmente em pulverização de instrumentos de medida. Considera-se que o trabalho foi capaz de apontar importantes direcionamentos para futuros estudos que envolvam as interfaces entre trabalho e família, de modo a contribuir com o desenvolvimento da área.

Palavras-chave: trabalho; família; conflito; equilíbrio.

\begin{abstract}
Work and family interfaces: Conceptual and empirical issues. The study of the interfaces between work and family can occur from two opposing perspectives: on one hand, we have the approach to work-family conflict, traditional line of research that considers that the demands arising from work and family are in general incompatible; on the other hand, we have a perspective that considers that the accumulation of occupational and family roles may be beneficial for individuals. This article aimed to characterize the two main theoretical perspectives that dominate research on work-family interfaces, highlighting how conceptual problems still present in both aspects translate into measurement problems, especially in the spraying of measurement instruments. It is considered that the study was able to point out important directions for future researches involving the interfaces between work and family, so as to contribute to the development of the area.
\end{abstract}

Keywords: work; family; conflict; equilibrium.

\section{Resumen}

Las interfaces entre trabajo y familia: Aspectos conceptuales y empíricos. El estudio de las interrelaciones entre el trabajo y la familia se puede producir a partir de dos perspectivas opuestas: por un lado, está el conflicto trabajo-familia, tradicional línea de investigación que considera que las demandas del trabajo y de la familia son incompatibles; Por otra parte, está la perspectiva que considera que la acumulación de papeles profesionales y familiares puede ser beneficiosa para los individuos. Este artículo tiene como objetivo caracterizar las dos principales perspectivas teóricas que dominan la investigación sobre las interfaces trabajo-familia, destacando como problemas conceptuales aún presentes en ambas vertientes se traducen en problemas de medición, especialmente en pulverización de instrumentos de medida. Se considera que el trabajo fue capaz de señalar algunas direcciones importantes para futuros estudios relacionados con las interfaces entre el trabajo y la familia.

Palabras clave: trabajo; familia; conflicto; equilibrio. 
Ao longo do percurso da investigação científica acerca das relações estabelecidas entre o trabaIho e a família, é possível identificar a existência de duas perspectivas distintas: de um lado, encontra-se a linha mais tradicional de pesquisa que tem como foco principal os efeitos negativos oriundos do acúmulo dos papéis familiares e ocupacionais (Greenhaus \& Beutell, 1985); de outro lado, encontra-se uma perspectiva mais recente (e ainda em fase de consolidação) que se caracteriza por apresentar uma visão mais otimista no que se refere ao engajamento em múltiplos papéis (Barnett \& Hyde, 2001). Tendo em vista que as concepções negativas e positivas das interfaces entre o trabalho e a família não são necessariamente excludentes (Matias \& Fontaine, 2012), trabalhos que integrem as duas visões têm sido apontados na literatura como uma alternativa com grande potencial para agregar valor à compreensão da complexa dinâmica que une os mundos do trabaIho e da família (Allen, Cho, \& Meier, 2014; Barnett \& Gareis, 2006; McMillan, Morris, \& Atchley, 2011).

Diante disso, o presente artigo tem o objetivo de caracterizar as duas principais perspectivas teóricas que dominam a pesquisa sobre as interfaces trabalho-família, destacando como problemas conceituais ainda presentes em ambas as vertentes se traduzem em problemas de mensuração, especialmente em pulverização de instrumentos de medida. Tal análise, tomando como referência estudos conduzidos no contexto brasileiro, busca embasar sugestões de desafios a serem enfrentados pelos pesquisadores que se dedicam a esse campo.

\section{Interfaces entre o trabalho e a família: a perspectiva negativa}

A partir da premissa básica de que a energia humana seria um recurso finito e escasso e que quanto mais energia fosse dedicada a um papel social menos dela estaria disponível para outro(s), desenvolveu-se uma visão de que pessoas, e em especial as mulheres, que acumulassem papéis - como os de esposas, mães e trabalhadoras - teriam maior tendência a vivenciar casamentos conturbados, a ter filhos com sentimentos de abandono e rejeição e, ainda, a ter mais problemas de sua saúde física e mental. Em 1985, Greenhaus e Beutell propõem o conceito de conflito trabalho-família para representar essas dificuldades de conciliação dos papéis, definindo-o como uma forma de conflito entre papéis no qual as pressões advindas do trabalho e da família são, de alguma forma, mutuamente incompatíveis.
Apesar de tal definição geral ser amplamente aceita e difundida, ela, por si só, não é suficiente para evidenciar a complexidade que cerca o construto. De fato, no mesmo trabalho em que tal definição é proposta, os autores reconhecem a existência de três elementos distintos que podem estar na origem da percepção de conflito entre trabalho e família: tempo, tensão e comportamento.

No primeiro caso, parte-se do pressuposto de que o tempo é um recurso finito e que gastá-lo em atividades de um domínio (trabalho; família) impede que ele seja destinado ao cumprimento de atividades de outra esfera da vida (família; trabalho). No segundo tipo de conflito, é a tensão gerada por um domínio que passa a ser a fonte de dificuldades para o bom desempenho em outro domínio. Por fim, o conflito baseado no comportamento emerge quando padrões comportamentais específicos de um papel são incompatíveis com as expectativas comportamentais para outro papel, e o indivíduo, por alguma razão, não consegue adaptar, de forma satisfatória, o seu comportamento a cada contexto solicitado (Greenhaus \& Beutell, 1985).

Para uma compreensão ainda mais completa do conflito, é preciso levar em consideração, também, que se trata de um fenômeno de caráter bidirecional, envolvendo a interferência do trabalho na família (ITF) e a interferência da família no trabalho (IFT). De acordo com Netemeyer, Boles e McMurrian (1996), a primeira direção (ITF) pode ser definida como um tipo de conflito entre papéis no qual as demandas de trabalho, além do tempo dedicado a ele e da tensão gerada por ele, interferem no desempenho das responsabilidades familiares. Já a direção oposta (IFT) se refere a um tipo de conflito entre papéis no qual as demandas da família, assim como o tempo dedicado a ela e a tensão gerada por ela, interferem no desempenho das responsabilidades ocupacionais. A Figura 1 apresenta os principais elementos presentes no modelo teórico do conflito trabalho-família.

Embora o caráter multidimensional e bidirecional do conflito trabalho-família tenha sido reconhecido desde os estudos teóricos iniciais, grande parte das medidas elaboradas para mensurar o fenômeno não acompanhou esse desenvolvimento conceitual, falhando em capturar as duas direções, em contemplar as três formas do conflito e/ou em alcançar boa qualidade psicométrica (Herst, 2003; Mesmer-Magnus \& Viswesvaran, 2005). A Tabela 1 sintetiza algumas das medidas até então propostas para a mensuração do fenômeno. 


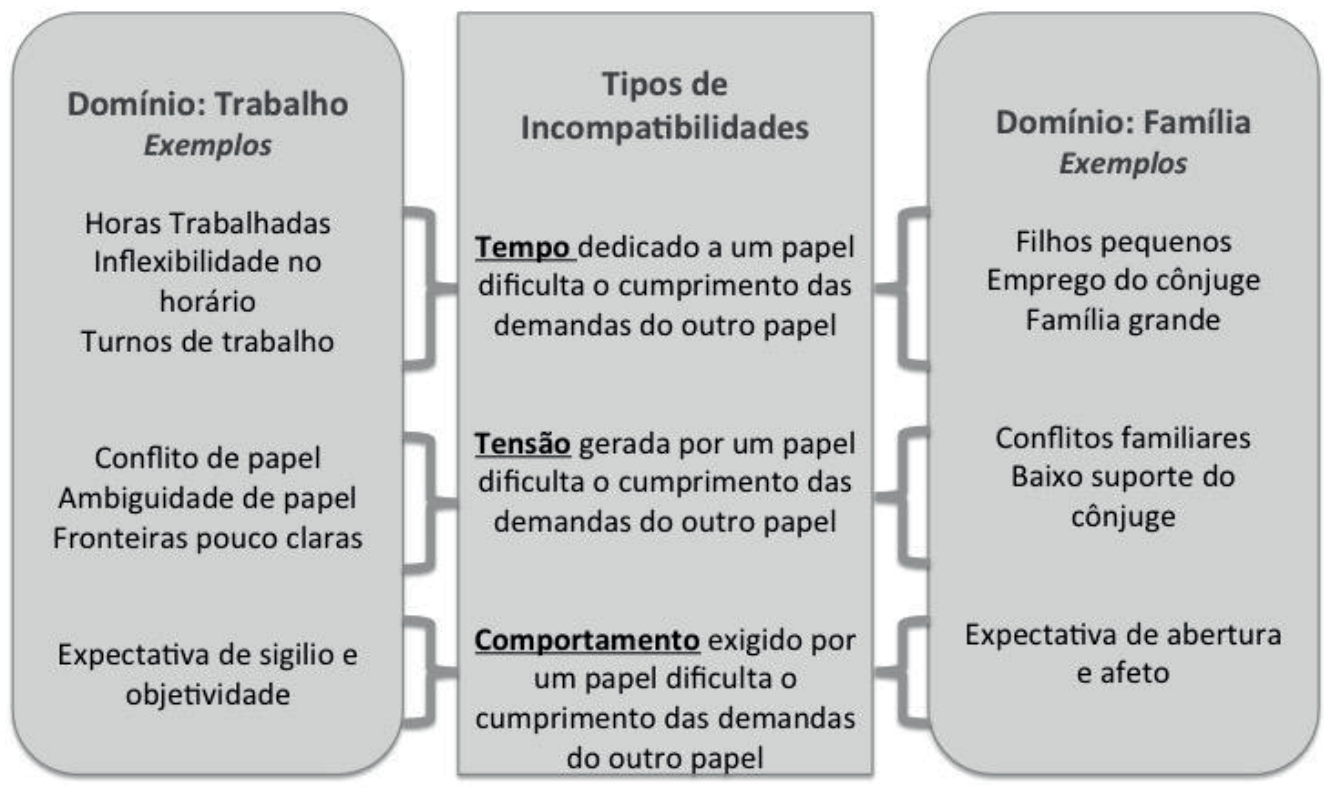

Figura 1. Modelo das Incompatibilidades entre Papéis Relacionados ao Trabalho e à Família.

Nota: Adaptado de Greenhaus e Beutell (1985).

Dentre as medidas existentes, a desenvolvida por Carlson et al. (2000) tem sido apontada como a que melhor satura o modelo teórico do conflito trabaIho-família e, ainda, a que apresenta melhores indicadores psicométricos (Lim, Morris, \& McMillan, 2011; Matthews, Kath, \& Barnes-Farrel, 2010). Para a sua construção, os autores conduziram três estudos. $O$ primeiro deles consistiu no levantamento e seleção de itens de escalas pré-existentes, seguido da análise de juízes para assegurar a validade de conteúdo dos mesmos, e da condução de Análise Fatorial Exploratória (AFE). Do total de 31 itens apresentados aos juízes, 20 permaneceram e foram aplicados em uma amostra de 390 pessoas que trabalhavam em uma agência governamental americana. Os resultados desse primeiro estudo apontaram a existência de três fatores, não representando, portanto, de forma satisfatória nenhuma das dimensões propostas pelo modelo teórico.

Os autores partiram, então, para um segundo estudo, que consistiu na criação de 34 itens adicionais que visaram possibilitar uma melhor representação das seis dimensões. Uma nova versão do instrumento (com 54 itens) foi novamente avaliada pelos juízes e 30 deles selecionados para compor a escala final. Por fim, o último estudo teve o objetivo de refinar e validar a escala resultante dos dois estudos anteriores, culminando na versão definitiva da escala, composta por 18 itens distribuídos em seis dimensões $\left(X^{2}=237,40 ; C F I=\right.$ 0,95; RMSEA = 0,06).

Uma vez publicada, a medida de Carlson et al. (2000) passou a ser amplamente adotada em estudos que se dedicam à apreensão do conflito trabalho-família ao redor do mundo (e.g.: Aguiar, 2016; Carr, Boyar, \& Gregory, 2008; Chambel, 2014; Li, Lu, \& Zhang, 2013; Lim et al, 2011; Sulea, Virgã, \& Galben, 2010). Chambel (2014), por exemplo, adotou uma versão traduzida da medida para a realidade portuguesa enquanto $\mathrm{Li}$ et al (2013) utilizaram-na para a mensuração do conflito entre chineses. Já Sulea et al (2010) a traduziram para aplicação entre romenos e Lim et al (2011) para uso na Coréia. Nos dois últimos casos mencionados, as análises psicométricas conduzidas indicaram que o modelo com seis fatores correlacionados (congruente com a proposta Carlson et al, 2000) foi o mais adequado para descrever os dados obtidos. Já nos dois primeiros casos mencionados, contudo, a estrutura original não obteve respaldo empírico, restringindo-se a soluções de dois fatores. Chambel (2014), inclusive, dedica parte do seu trabalho para apresentar os resultados referentes às qualidades psicométricas alcançadas pela escala em seu estudo e conclui que, por meio de análises fatoriais, é possível afirmar que o fenômeno é melhor representado na amostra em questão por dois únicos fatores: conflito trabalho-família e conflito família-trabalho. 
Tabela 1. Medidas do Conflito Trabalho-Família
Autores
Principais características da medida

\begin{abstract}
Burke, Weir \& DuWors
(1979)

Pleck, Staines \& Lang

(1980)

Bohen \& Viveros-Long

Mensura o grau em que o cônjuge percebe o trabalho do parceiro impactando negativamente na vida familiar.

Medida em formato de survey que determina a frequência e o tipo do conflito trabalho-família encontrado em diversas estruturas familiares.

Descreve a frequência com que os participantes experienciam stress e tensão relacionada à combinação entre trabalho e família. A medida acessa múltiplos aspectos do conflito, como ambiguidade de normas, insuficiência de recursos, incongruência entre personalidade e normas sociais etc.
\end{abstract}

Kopelman, Greenhaus \&

Connoly (1983)

Wiley (1987)

Small \& Riley (1990)

Gutek, Searle \& Kepla

Bacharach, Bamberger \& Conley (1991)

Thomas \& Gasnter

Stephens \& Sommer

Netemyer, Boles, \& McMurrian (1996)

Sanders, Lengnick-Hall, Lengnick-Hall, \& SteeleClapp (1998)

Carlson, Kacmar \& Williams (2000)

Carlson \& Frone (2003)

Composta por oito itens elaborados para acessar a extensão do conflito entre papéis que ocorre entre o trabalho e a família, considerando apenas uma direção.

Trabalha com quatro fatores: a. conflito trabalho/indivíduo; b. conflito trabalho/família; c. conflito família/trabalho; d. sobrecarga de papel.

Mensura o extravasamento entre demandas de trabalho e quatro tipos de demandas pessoais: a. lazer; $b$. afazeres domésticos; c. cônjuge; d. paternidade/maternidade.

Primeira medida a considerar as duas direções do conflito: demandas familiares interferindo nas responsabilidades ocupacionais e vice-versa.

Acessa a extensão do conflito entre responsabilidades do trabalho e responsabilidades domésticas, considerando apenas a direção da interferência do trabalho na família.

Mede a percepção de controle sobre aspectos relacionados a responsabilidades e demandas que podem contribuir para o conflito, como jornada de trabalho e flexibilidade de horário.

Os itens descrevem conflitos que se originam no ambiente de trabalho e podem afetar a família. Considera apenas uma direção (interferência do trabalho na família), mas a apreende a partir de três dimensões distintas: conflito baseado no tempo, na tensão e no comportamento.

Utiliza duas diferentes subescalas para acessar tanto a interferência do trabalho na família quanto a interferência da família no trabalho.

Composta por 50 itens elaborados para acessar as atitudes do trabalhador no que diz respeito ao manejo da interface entre sua carreira e sua família.

Primeira escala que une a bidirecionalidade e a multidimensionalidade do conflito trabalho-família. A medida é composta por seis subescalas, que surgem a partir das combinações entre duas direções (interferência do trabalho na família e interferência da família no trabalho) e as três formas de conflito (baseado no tempo, na tensão e no comportamento).

Considera que o conflito deve ser divido entre interno e externo. A medida é composta por quatro dimensões: a. interferência interna do trabalho na família; b. interferência externa do trabalho na família; c. interferência interna da família no trabalho; d. interferência externa da família no trabalho.

Matthews, Kath, \& Representa uma versão reduzida da escala de Carlson et al (2000), composta por seis itens: um para cada combinação entre Barnes-Farrel (2010) natureza e direção do fenômeno.

Haslam, Filus, Desenvolvida para ser utilizada exclusivamente com pais de crianças até 12 anos de idade. É composta por 10 itens e Morawska, Sanders, \& Fletcher (2014)

Breyer \& Bluemke (2016) apreende a bidirecionalidade do conflito trabalho-família.

Consiste em questionário composto por quatro itens, sendo dois para a mensuração da interferência do trabalho na família e dois para a mensuração da interferência da família no trabalho. Os itens têm sido usados desde 2002 em diversos idiomas pelo International Social Survey Programme (ISSP).

Nota: Elaborado a partir de Carlson e Frone (2003), Carlson, Kacmar e Williams (2000), Fields (2002), Haslam, Filus, Morawska, Sanders \& Fletcher (2014), Herst (2003), Matthews, Kath \& Barnes-Farrel (2010), Netemeyer, Boles \& McMurrian (1996) e Breyer \& Bluemke (2016). 
Dentre as medidas existentes, a desenvolvida por Carlson et al. (2000) tem sido apontada como a que melhor satura o modelo teórico do conflito trabaIho-família e, ainda, a que apresenta melhores indicadores psicométricos (Lim, Morris, \& McMillan, 2011; Matthews, Kath, \& Barnes-Farrel, 2010). Para a sua construção, os autores conduziram três estudos. $O$ primeiro deles consistiu no levantamento e seleção de itens de escalas pré-existentes, seguido da análise de juízes para assegurar a validade de conteúdo dos mesmos, e da condução de Análise Fatorial Exploratória (AFE). Do total de 31 itens apresentados aos juízes, 20 permaneceram e foram aplicados em uma amostra de 390 pessoas que trabalhavam em uma agência governamental americana. Os resultados desse primeiro estudo apontaram a existência de três fatores, não representando, portanto, de forma satisfatória nenhuma das dimensões propostas pelo modelo teórico.

Os autores partiram, então, para um segundo estudo, que consistiu na criação de 34 itens adicionais que visaram possibilitar uma melhor representação das seis dimensões. Uma nova versão do instrumento (com 54 itens) foi novamente avaliada pelos juízes e 30 deles selecionados para compor a escala final. Por fim, o último estudo teve o objetivo de refinar e validar a escala resultante dos dois estudos anteriores, culminando na versão definitiva da escala, composta por 18 itens distribuídos em seis dimensões $\left(X^{2}=237,40 ; C F I=\right.$ 0,95; RMSEA $=0,06$ ).

Uma vez publicada, a medida de Carlson et al. (2000) passou a ser amplamente adotada em estudos que se dedicam à apreensão do conflito trabalho-família ao redor do mundo (e.g.: Aguiar, 2016; Carr, Boyar, \& Gregory, 2008; Chambel, 2014; Li, Lu, \& Zhang, 2013; Lim et al, 2011; Sulea, Virgã, \& Galben, 2010). Chambel (2014), por exemplo, adotou uma versão traduzida da medida para a realidade portuguesa enquanto Li et al (2013) utilizaram-na para a mensuração do conflito entre chineses. Já Sulea et al (2010) a traduziram para aplicação entre romenos e Lim et al (2011) para uso na Coréia. Nos dois últimos casos mencionados, as análises psicométricas conduzidas indicaram que o modelo com seis fatores correlacionados (congruente com a proposta Carlson et al, 2000) foi o mais adequado para descrever os dados obtidos. Já nos dois primeiros casos mencionados, contudo, a estrutura original não obteve respaldo empírico, restringindo-se a soluções de dois fatores. Chambel (2014), inclusive, dedica parte do seu trabalho para apresentar os resultados referentes às qualidades psicométricas alcançadas pela escala em seu estudo e conclui que, por meio de análises fatoriais, é possível afirmar que o fenômeno é melhor representado na amostra em questão por dois únicos fatores: conflito trabalho-família e conflito família-trabalho.

No Brasil, Aguiar (2016) dedicou-se ao processo de tradução, adaptação e validação da medida para uso em- trabalhadores brasileiros. Após a aplicação do instrumento traduzido em uma ampla amostra de trabalhadores e posterior a condução de procedimentos estatísticos que envolveram análises fatoriais exploratórias e confirmatórias, a autora não conseguiu sustentar empiricamente uma estrutura fatorial similar à defendida por Carlson et al. (2000).

Uma primeira dificuldade referiu-se às sobreposições das naturezas tempo e tensão nas duas direções do conflito, representando a dificuldade em se diferenciar empiricamente essas duas fontes de origem do conflito (evidenciadas por correlações extremamente elevadas - superiores a 0,80). Através da retomada do trabalho original de Greenhaus e Beutell (1985), é possível perceber que tal dificuldade de diferenciação não é exclusivamente empírica, uma vez que a própria descrição conceitual da natureza temporal já prevê sua estreita relação com o conflito baseado na tensão. De acordo com os autores, o conflito baseado no tempo pode assumir duas formas: 1. A pressão para dedicar muito tempo a um dos domínios (trabalho; família) pode tornar fisicamente impossível o cumprimento das expectativas advindas do outro domínio (família; trabaIho) ou 2. Essa mesma pressão para dedicar tempo a um domínio (trabalho; família) pode gerar uma preocupação que permanece presente mesmo quando o indivíduo está fisicamente presente em outro domínio (família; trabalho). Fica claro, portanto, que a segunda forma assumida pelo conflito baseado no tempo estará, muito provavelmente, acompanhada do sentimento de estresse e/ou ansiedade, que são os elementos constitutivos do conflito baseado na tensão.

Além das sobreposições entre as bases de tensão e tempo, algumas fragilidades foram verificadas também na base comportamental do conflito trabalho-família. Entre elas, destaca-se a forte correlação entre as duas direções do conflito baseado no comportamento $(r=0,846, p<0,001)$. Ademais, uma análise de consistência interna de cada uma das dimensões revela resultados insatisfatórios $(a=0,45$ e 0,61 para a ITF baseada no comportamento e para a IFT baseada no comportamento, respectivamente). 
Da mesma forma como já havia ocorrido previamente em alguns outros países (eg.: Chambel, 2014; Li et al., 2013), no contexto brasileiro, a solução fatorial que se revelou mais adequada restringiu-se a uma estrutura bidimensional que contempla o fenômeno a partir de suas duas direções: interferência do trabalho na família ( 8 itens, $a=0,87$ ) e interferência da família no trabalho (8 itens, $a=0,80$ ).

Em síntese, percebe-se a inconsistência dos resultados empíricos, o que aponta para a necessidade de se repensar o modelo teórico clássico e hegemonicamente utilizado de Greenhaus e Beutell (1985). Ao que parece, a existência de duas direções para o conflito está posta e é facilmente identificada empiricamente; entretanto, as naturezas que estão na base do conflito (tempo, tensão e comportamento) não têm sido percebidas de formas significativamente distintas pelos indivíduos em todas as realidades em que o modelo foi testado. Reconhece-se, entretanto, que apenas através de novos estudos, conduzidos em diversas localizações geográficas, será possível concluir acerca da pertinência de se propor uma reformulação do modelo teórico até então amplamente adotado e, consequentemente, acerca da estrutura fatorial que permita uma mensuração adequada do fenômeno conflito trabalho-família.

\section{Interfaces entre o trabalho e a família: a perspectiva positiva}

Em contraposição à perspectiva do conflito trabaIho-família, surge na literatura uma abordagem predominantemente otimista no que se refere às consequências do engajamento do indivíduo em múltiplos papéis. Essa abordagem passa a defender a possibilidade de tal acúmulo trazer benefícios tanto para mulheres quanto para os homens (e.g., Barnett \& Hyde, 2001; Carlson, Kacmar, Wayne, \& Grzywacz, 2006; Frone, 2003; Wayne, Grzywacz, Carlson, \& Kacmar, 2007). Apesar do crescente interesse por tal perspectiva positiva, o corpo de conhecimento sobre os benefícios dos múltiplos papéis ainda é extremamente reduzido quando comparado com a produção existente sobre os conflitos e potenciais prejuízos causados pelo acúmulo de diversos papéis e, em especial, dos familiares e ocupacionais (Aryee, Srinivas, \& Tan, 2005; Chambel \& Santos, 2009; Greenhaus \& Powell, 2006).

De acordo com Hanson, Hammer e Colton (2006), embora o conhecimento sobre a perspectiva positiva seja de fundamental importância para o campo de estudo das relações entre trabalho e família, a produção de trabalhos científicos nessa área ainda tem progredido de forma muito lenta. Segundo os autores, há uma forte necessidade de investimentos voltados para três aspectos fundamentais: 1. desenvolvimento de um arcabouço teórico sólido; 2. maior clarificação conceitual e empírica do construto; 3. elaboração de ferramentas de mensuração apropriadas.

No que diz respeito ao arcabouço teórico que tem embasado o entendimento sobre o lado positivo da interação trabalho-família até então, a teoria que tem obtido maior destaque é a proposta por Barnett e Hyde (2001), denominada de teoria expansionista - assim chamada por considerar que a energia humana é um recurso expansível, e não limitado e escasso como considerado pela perspectiva negativa da interação entre trabalho e família. O pressuposto básico da teoria é que múltiplos papéis não são necessariamente nocivos ao indivíduo; ao contrário, estar engajado em mais de um papel social é, via de regra, benéfico, gerando reflexos positivos para a saúde mental, para a saúde física e até mesmo para a qualidade dos relacionamentos conjugais. Entretanto, para que tais efeitos positivos sejam possíveis, a teoria expansionista reconhece que existem determinados processos e condições que precisam ser satisfeitos (Barnett \& Hyde, 2001).

Em relação aos processos que têm o potencial de contribuir para os efeitos positivos derivados do engajamento em múltiplos papéis, as autoras identificaram oito fatores que são capazes de afetar a magnitude dessa relação, conforme sintetizado na Tabela 2.

Embora os processos mencionados sejam os apontados por Barnett e Hyde (2001), as autoras não desconsideram a possibilidade de haver outros elementos capazes de potencializar os efeitos positivos do acúmulo dos papéis de trabalhador(a) e membro participativo de uma família. E, além desses processos moderadores, existem pelo menos duas condições básicas que precisam ser supridas: a primeira refere-se à existência de um limite aceitável em relação ao número de papéis nos quais o indivíduo se engaja e à carga de demandas que surge de cada um deles; e a segunda condição estabelece que a qualidade da experiência vivenciada no(s) papel(is) é mais importante que o número ou tempo dedicado a ele(s) (Barnett \& Hyde, 2001). Um exemplo referente à primeira condição seria o caso de uma pessoa que é casada, tem filhos, assume um cargo de chefia na empresa na qual trabalha e ainda acumula os papéis 
de estudante e responsável por cuidar dos pais idosos. Nesse caso, muito provavelmente a elevada quantidade de papéis que foram assumidos simultaneamente acarretam uma sobrecarga de demanda que se torna prejudicial para o indivíduo. Em relação à segunda condição, pode-se citar como exemplo ilustrativo o fato de que a vida familiar de um indivíduo não se beneficiará da sua inserção no mercado do trabalho se suas experiências no emprego forem predominantemente negativas (em decorrência de fatores como elevada cobrança, assédio moral, conflitos com colegas de trabalho, entre outros).

Tabela 2. Processos Apontados como Potenciais Facilitadores para os Efeitos Positivos Oriundos do Engajamento em Múltiplos Papéis

\begin{tabular}{|c|c|}
\hline Processo & Explicação \\
\hline Efeito barreira & $\begin{array}{l}\text { Sugere que os efeitos negativos do estresse ou fracasso em um papel podem ser compensadas pelo sucesso e } \\
\text { satisfação obtidos em outro papel. }\end{array}$ \\
\hline $\begin{array}{l}\text { Incremento da } \\
\text { renda familiar }\end{array}$ & $\begin{array}{l}\text { Identifica que a renda gerada por um casal de dupla-renda tem o potencial de beneficiar concretamente } \\
\text { a qualidade de vida da família e, ainda, reduzir a possível ansiedade e insegurança sentidas quando existe } \\
\text { apenas um responsável pela manutenção financeira da família. }\end{array}$ \\
\hline Suporte social & $\begin{array}{l}\text { Considera que múltiplos papéis potencializam as chances de se obter suporte social, o que, por sua vez, } \\
\text { aumenta o bem-estar dos indivíduos. }\end{array}$ \\
\hline Oportunidades de sucesso & $\begin{array}{l}\text { Aponta que o engajamento em diversos papéis disponibiliza diferentes oportunidades para experiências de } \\
\text { sucesso e para o desenvolvimento de um senso de autoconfiança e autoeficácia. }\end{array}$ \\
\hline Expansão de referências & $\begin{array}{l}\text { Considera que pessoas que combinam múltiplos papéis têm mais oportunidades de obter informações e, } \\
\text { consequentemente, de expandir suas perspectivas e pontos de vista sobre as situações com as quais se } \\
\text { deparam. }\end{array}$ \\
\hline Aumento da autoavaliação & $\begin{array}{l}\text { Sugere que pessoas com representações cognitivas mais complexas sobre si mesmas tendem a desenvolver } \\
\text { autoavaliações mais positivas. }\end{array}$ \\
\hline Similaridade de Experiências & $\begin{array}{l}\text { No caso de pessoas casadas, quando os cônjuges acumulam atividades de trabalho e família, as experiências } \\
\text { do casal se tornam mais similares, facilitando a comunicação e promovendo maior qualidade do } \\
\text { relacionamento. }\end{array}$ \\
\hline Ideologia de gênero & $\begin{array}{l}\text { Aponta que extensão na qual o indivíduo possui atitudes tradicionais ou liberais em relação aos papéis sociais } \\
\text { de homens e mulheres pode influenciar no benefício (ou malefício) do acúmulo de papéis. Pessoas com } \\
\text { ideologias mais liberais sobre os gêneros tendem a se beneficiar mais da combinação dos papéis ocupacionais } \\
\text { e familiares. }\end{array}$ \\
\hline
\end{tabular}

Nota: Adaptado de Barnett e Hyde (2001).

Outro ponto que tem trazido alguns desafios para essa área de estudo se refere às diversas nomenclaturas e definições adotadas por diferentes pesquisadores para se referirem à relação positiva entre o trabalho e a família. Rótulos como enriquecimento trabalho-familia, facilitação trabalho-familia, valorização trabalho-família e extravasamento positivo entre trabalho e familia têm sido utilizados para falar das vantagens da participação simultânea nas esferas ocupacional e familiar. Enquanto alguns autores consideram que tais nomenclaturas devem ser encaradas como sinônimos para representar o lado positivo da relação (e.g., Frone, 2003; Greenhaus \& Powell, 2006; McNall, Masuda, \& Nicklin, 2010), outros defendem que o enriquecimento, a facilitação, a valorização e o extravasamento positivo entre trabalho e família são conceitos distintos entre si (e.g.,
Carlson et al., 2006; Hanson et al., 2006; Steenbergen, Ellemers, \& Mooijaart, 2007). A Tabela 3 apresenta as principais nomenclaturas adotadas na literatura e suas respectivas definições.

A partir das diversas definições apresentadas, o que se nota é que todas elas apresentam um núcleo comum - que se refere à capacidade das experiências vividas em um domínio (trabalho; família) gerarem benefícios diretos e significativos para o outro domínio (família; trabalho) - o que as coloca sob um mesmo "guarda-chuva" conceitual. De fato, a grande proximidade entre os construtos pode ser reforçada por meio da análise das medidas até então propostas para a mensuração de cada um desses fenômenos. A Tabela 4 apresenta as características das principais medidas existentes na área. 
Tabela 3. Nomenclaturas e Definições das Relações Positivas entre Trabalho e Família

\begin{tabular}{cl}
\hline Conceito & \multicolumn{1}{c}{ Definição } \\
\hline Facilitação & Refere-se à extensão na qual a participação em um papel é melhorada/facilitada em virtude da participação \\
(Facilitation) & em outro papel (Wayne et al., 2004). \\
Valorização & Representa a aquisição de recursos e experiências em um papel que são benéficos para os indivíduos no \\
(Enhancement) & desempenho de desafios em outro papel (Sieber, 1974 citado por Fisher, Bulger \& Smith, 2009). \\
Extravasamento positivo & Refere-se a experiências em um domínio (afetos, habilidades, valores e comportamentos) sendo transferidas \\
(Positive Spillover) & para outro domínio de forma que as experiências desses dois domínios se tornem mais próximas (Edwards \& \\
& Rothbard, 2000). \\
& Refere-se à extensão na qual as experiências em um papel melhoram a qualidade de vida em outro papel, \\
Enriquecimento & ocorrendo quando recursos gerados em um domínio (como habilidades, flexibilidade, capital social e material \\
(Enrichment) & etc) se tornam responsáveis por melhorar o desempenho e/ou o estado afetivo em outro domínio (Greenhaus \\
& \& Powell, 2006).
\end{tabular}

Tabela 4. Medidas do Enriquecimento, Valorização, Facilitação e Extravasamento Positivo entre Trabalho e Família e Suas Respectivas Dimensões

\begin{tabular}{|c|c|}
\hline \multirow{2}{*}{ Conceitos e autores } & Dimensionalida \\
\hline & Trabalho x Família \\
\hline \multirow{7}{*}{$\begin{array}{c}\text { Enriquecimento } \\
\text { Carlson } \\
\text { et al. (2006) } \\
\text { Kacmar, Crawford, Carlson, } \\
\text { Ferguson, \& Whitten (2014) }\end{array}$} & CAPITAL: \\
\hline & O envolvimento no trabalho promove aumento de recursos \\
\hline & psicológicos que ajudam o indivíduo a ser um melhor \\
\hline & membro para a sua família. \\
\hline & AFETO: \\
\hline & $\begin{array}{c}\text { O envolvimento no trabalho resulta em um estado } \\
\text { emocional positivo que ajuda o indivíduo a ser um melhor } \\
\text { membro para a sua família }\end{array}$ \\
\hline & $\begin{array}{l}\text { DESENVOLVIMENTO: } \\
\text { O envolvimento no trabalho leva à aquisição de }\end{array}$ \\
\hline
\end{tabular}

habilidades, conhecimentos, comportamentos e/ou pontos de vista que ajudam o indivíduo a ser um melhor membro para a sua família.

Extravasamento positivo Hanson et al. (2006)

Facilitação

Steenbergen et al. (2007)

Valorização

Fischer et al. (2009)
BASEADO NO AFETO:

O transbordamento do estado emocional originado no trabalho para a esfera familiar.

BASEADO NO COMPORTAMENTO:

Possibilidade de aproveitar comportamentos e habilidades aprendidas no trabalho para melhorar o desempenho na esfera familiar.

BASEADO NOS VALORES:

Uso de valores e princípios adquiridos/ desenvolvidos no trabalho para melhorar o desempenho na esfera familiar.

BASEADA NO TEMPO:

O tempo devotado ao trabalho facilita o manejo eficiente do tempo nas atividades familiares. BASEADA NA ENERGIA:

A energia obtida no trabalho facilita o cumprimento das demandas familiares.

BASEADA NO COMPORTAMENTO:

O comportamento aprendido/requerido no trabalho facilita o cumprimento de demandas familiares. PSICOLÓGICA:

Capacidade de analisar os problemas relacionados à família sob diferentes perspectivas em função da participação no domínio ocupacional.

VALORIZAÇÃO TRABALHO-FAMÍLIA:

Aquisição de recursos e experiências no ambiente ocupacional que são benéficos para desempenho frente aos desafios familiares.
Família $\mathrm{x}$ Trabalho

EFICIÊNCIA:

O envolvimento na família promove um senso de foco que ajuda o indivíduo a ser um trabalhador melhor.

AFETO:

O envolvimento na família resulta em uma atitude ou estado emocional positivo que ajuda o indivíduo a ser um trabalhador melhor. DESENVOLVIMENTO:

O envolvimento na família leva à aquisição de habilidades, conhecimentos, comportamentos e/ou pontos de vista que ajudam o indivíduo a ser um trabalhador melhor.

BASEADO NO AFETO:

O transbordamento do estado emocional originado na família para a esfera ocupacional. BASEADO NO COMPORTAMENTO

Possibilidade de aproveitar comportamentos e habilidades aprendidas na família para melhorar o desempenho na esfera ocupacional.

BASEADO NOS VALORES:

Uso de valores e princípios adquiridos/ desenvolvidos na família para melhorar o desempenho na esfera ocupacional.

BASEADA NO TEMPO:

O tempo devotado à família facilita o manejo eficiente do tempo nas atividades de trabalho. BASEADA NA ENERGIA:

A energia obtida na família facilita o cumprimento das demandas ocupacionais. BASEADA NO COMPORTAMENTO:

O comportamento aprendido/requerido na família facilita o cumprimento de demandas ocupacionais. PSICOLÓGICA:

Capacidade de analisar os problemas relacionados ao trabalho sob diferentes perspectivas em função da participação no domínio familiar.

VALORIZAÇÃO FAMÍLIA-TRABALHO:

Aquisição de recursos e experiências no ambiente familiar que são benéficos para desempenho frente aos desafios ocupacionais. 
Com base na revisão das principais medidas existentes na área, nota-se que há um predomínio de escalas multidimensionais e que, em muitos casos, uma ou mais dimensões dos diferentes instrumentos estão claramente sobrepostas. A dimensão comportamental, por exemplo, surge nos conceitos de facilitação e de extravasamento positivo com definições extremamente próximas que se referem ao potencial de os comportamentos aprendidos em uma das esferas contribuir para o aprimoramento do desempenho em outra esfera da vida. De forma similar, a dimensão afetiva é contemplada nos conceitos de enriquecimento e também de extravasamento positivo, referindo-se à transposição de estados emocionais de um domínio para o outro. Cabe notar, ainda, a presença de dimensões que não são nomeadas da mesma forma nos diferentes construtos, mas guardam grande proximidade entre si quando se observa as suas definições. É o que acontece, por exemplo, com as dimensões capital e psicológica dos conceitos de enriquecimento e facilitação, respectivamente: ambas referem-se a um ganho de recursos psicológicos em função da participação em um domínio que contribui para um melhor desempenho frente a situações enfrentadas em outro domínio.

O quadro apresentado até aqui evidencia a grande confusão conceitual e empírica que cerca esse campo de estudo, sendo possível concluir que a pluralidade de rótulos e de instrumentos não significa, necessariamente, que objetos diferentes estejam sendo mensurados. Ao contrário, o enriquecimento, a valorização, a facilitação, assim como o extravasamento positivo entre o trabalho e a família parecem caracterizar e nomear um fenômeno comum, que pode ser compreendido como as diferentes formas através das quais o acúmulo dos papéis de trabalhador e de membro ativamente participativo de uma família contribuem para um melhor desempenho em ambos os domínios.

Considerando que as nomenclaturas enriquecimento, facilitação, valorização e extravasamento positivo trabalho-família deveriam ser compreendidas a partir de um espectro único, Aguiar e Bastos (2018) propuseram uma medida integrada para a mensuração da interface positiva entre o trabalho e a família. Em uma primeira fase, os itens das medidas previamente identificadas na literatura foram traduzidos e adaptados para a língua portuguesa e, em seguida, aplicados em uma amostra de trabalhadores brasileiros. A partir de procedimentos de análise que envolveram, predominantemente, análises fatoriais exploratórias e avaliação semântica dos conteúdos dos itens, foi possível propor uma primeira versão de um instrumento integrador, composto por 20 itens. Desse total de itens, oito foram derivados da escala de extravasamento positivo trabalho-família, seis da escala de enriquecimento trabalho-família, quatro da escala de facilitação trabalho-família e dois da escala de valorização trabaIho-família. Nota-se, portanto, que todas as medidas foram contempladas e capazes de contribuir, em algum grau, para a elaboração da proposta de um instrumento único e integrador, reforçando a ideia de que todas as nomenclaturas e respectivas definições localizadas na literatura podem ser compreendidas a partir de um mesmo construto - a interface positiva entre o trabaIho e a família.

Uma vez finalizada a versão inicial, um novo estudo empírico foi conduzido com uma nova amostra de trabalhadores brasileiros com o objetivo de avaliar a estrutura fatorial mais adequada para o fenômeno. Por meio de novos procedimentos de análise, desta vez incluindo análises fatoriais exploratórias e confirmatórias, foram testadas diversas soluções fatoriais para a medida, que variaram de uma estrutura com cinco dimensões até uma solução unifatorial. Os resultados indicaram a impossibilidade de distinguir satisfatoriamente possíveis subdimensões que compõem a interface positiva entre o trabalho e a família, parecendo mais promissora a consideração de que a medida deve levar em consideração, apenas, as duas direções do fenômeno. Diante disso, os autores recomendam que a interface positiva entre o trabalho e a família passe a ser apreendida, no contexto brasileiro, a partir de duas grandes dimensões: interface positiva trabalho-família e interface positiva família-trabalho (Aguiar \& Bastos, 2018).

\section{Considerações finais}

Inquestionavelmente, as relações estabelecidas entre os contextos laboral e familiar têm sido alvo de crescente interesse de pesquisadores em diferentes localizações geográficas. As grandes transformações por que passou no mundo nas últimas décadas, com profundas reestruturações dos contextos e condições de trabalho, assim como nas estruturas e dinâmicas familiares, ampliam a relevância de se compreender, em diferentes contextos culturais, como demandas dessas duas importantes esferas da vida são articuladas e 
vivenciadas por cada indivíduo. Os impactos das formas como tais demandas são ou não articuladas certamente afetam o desempenho do indivíduo nos dois papéis. É interessante destacar que tal relevância fica ainda mais evidente quando os avanços tecnológicos estão permitindo, cada vez mais, que trabalho e família compartiIhem os mesmos espaços físico e temporal.

Apesar da relevância e a atualidade do estudo sobre as interfaces entre trabalho e família, pode-se verificar que a pesquisa, quer na vertente do conflito, quer na vertente positiva, encontra-se envolvida em problemas conceituais ainda não equacionados, o que se traduz em problemas na mensuração dos fenômenos. Tal situação não diferencia essas vertentes de pesquisa do que se verifica frente a todos os demais construtos no campo do Comportamento Organizacional, ou mesmo da Psicologia. Tem-se, aqui, mais um exemplo de fragmentação conceitual (especialmente na vertente positiva, com inúmeros nomes para rotular um fenômeno que parece ter uma natureza única) que se traduz em pulverização de medidas, de instrumentos que atrasam o próprio desenvolvimento do campo ao dificultar o processo cumulativo de produção de evidências empíricas.

Em ambas as perspectivas de pesquisa há problemas conceituais que precisam ser enfrentados, inclusive em estudos transculturais. Quando se trata do conflito trabalho família, parte-se de uma definição mais consensual do construto, cujo foco nos problemas, dificuldades e tensões geradas pelos dois papéis são reconhecidos como bidirecionais. Há, no entanto, um modelo que agrega as fontes principais do conflito que, apesar de ser largamente aceito, não tem encontrado validade empírica em instrumentos construídos e validados em diferentes países. $O$ ideal de parcimônia recomendaria, no atual estágio da pesquisa, não considerar tais dimensões para diferenciar as intensidades ou modalidades com que os indivíduos vivenciam o conflito entre o seu papel como trabalhador e como membro da família.

Da mesma forma, e talvez com maior intensidade, o lado positivo das relações entre o trabalho e a família revela problemas conceituais que se traduzem em problemas de mensuração. Enquanto conflito trabalho-família é um rótulo consensual na primeira vertente, aqui temos uma pulverização de rótulos cuja variação não encontra suporte até mesmo na definição teórica de cada conceito. Ou seja, quando se trata de estudar as interfaces positivas, um primeiro desafio é o de se aprofundar se os diferentes conceitos usados correspondem, de fato, a fenômenos distintos ou são rótulos distintos para um único fenômeno. Neste sentido, o estudo conduzido por Aguiar e Bastos (2018) aponta na direção de que os diferentes rótulos não se referem a fenômenos distintos. O presente trabalho sugere que novos estudos, especialmente de validade discriminante, devem ser conduzidos de modo a fortalecer as evidências e permitir, no futuro, a adoção de um conceito único e integrador para se fazer referência a esse tipo de interrelação entre os domínios familiar e ocupacional, denominado de interface positiva entre trabalho e família.

Em suma, considera-se que o presente trabalho, ao discutir como as duas perspectivas de se estudar as interfaces trabalho família ainda apresentam problemas conceituais que se traduzem em problemas de mensuração, aponta a necessidade de uma agenda de pesquisa que possa melhor solucionar tais problemas, incrementando as contribuições de tais estudos para compreender dinâmicas laborais e familiares. Certamente tal agenda de pesquisa deveria valorizar estudos transculturais - considerando diferenças nacionais ou mesmo regionais dentro de um mesmo país - que possam contribuir para o aperfeiçoamento dos modelos teóricos hoje disponíveis. Outro elemento importante desta agenda de pesquisa é levar em consideração características próprias dos contextos de trabalho (por exemplo, tipo de organização, modelo de gestão, natureza do trabalho) e das estruturas familiares (famílias nucleares, matrifocais, anaparentais, etc) de modo que possam identificar variáveis moderadoras que ajudem a verificar a generalidade dos modelos teóricos hoje disponíveis.

\section{Referências}

Aguiar, C.V. N. (2016). Interfaces entre o trabalho e a familia e os vínculos organizacionais: explorando a tríade família-trabalho-organização (Tese de Doutorado). Recuperado de http://repositorio.ufba.br/ri/ handle/ri/22618

Aguiar, C. V. N.; \& Bastos, A. V. B. (2018). Interface positiva trabalho e família: proposta de instrumento e evidências de validade. Revista de Avaliação Psicológica, 17(1), 48-58. doi: 10.15689/ ap.2017.1701.06.13175

Allen, T.D, Cho, E., \& Meier, L. L. (2014). Work-family boundary dynamics. Annual Review of Organizational Psychology and Organizational Behavior, 1, 99-121. doi: 10.1146/annurev-orgpsych-031413-091330

Aryee, S., Srinivas, E. S., \& Tan, H.H. (2005). Rhythms of life: Antecedentes and outcomes of work-family balance in employed parentes. Journal of Applied Psychology, 90(1), 132-146. doi: 10.1037/0021-9010.90.1.132

Barnett, R. C., \& Gareis, K. C. (2006). Role theory perspectives on work and family. In M. Pitt-Catsouphes, E. E. Kossek, \& S. Sweet (Orgs), 
Interfaces entre trabalho e família

The work and family handbook: Multi-disciplinary perspectives, methods, and approaches (pp. 209-221). Mahwah, NJ: Lawrence Erlbaum Associates.

Barnett, R. C., \& Hyde, J. S. (2001). Women, men, work, and family: An expansionist theory. American Psychologist, 56(10), 781-796. doi: 10.1037/0003-066X.56.10.781

Carlson, D. S., \& Frone, M. R. (2003). Relation of behavioral and psychological involvement to a new four-factor conceptualization of work-family interference. Journal of Business and Psychology, 17(4), 515-535. doi: 10.1023/A:1023404302295

Carlson, D. S, Kacmar, K. M., Wayne, J. H., \& Grzywacz, J. G. (2006). Measuring the positive side of the work-family interface: Development and validation of a work-family enrichment scale. Journal of Vocational Behavior, 68, 131-164. doi: 10.1016/j.jvb.2005.02.002

Carlson, D. S., Kacmar, K. M., \& Williams, L. J. (2000). Construction and initial validation of a multidimensional measure of work-family conflict. Journal of Vocational Behavior, 56, 249-276. doi: 10.1006/ jvbe.1999.1713

Carr, J. C., Boyar, S. L., \& Gregory, B. T. (2008). The moderating effect of work-family centrality on work-family conflict, organizational attitudes, and turnover behavior. Journal of Management, 34(2), 244-262. doi: $10.1177 \% 2 F 0149206307309262$

Chambel, M. J. (2014). A interface entre o trabalho e a família. In M. J. Chambel \& M. T. Ribeiro (Orgs.), A relação entre o trabalho e a família: do conflito ao enriquecimento (pp.3-8). Lisboa: RH Editora.

Chambel, M. J., \& Santos, M. V. (2009). Práticas de conciliação e satisfação no trabalho: mediação da facilitação do trabalho na família. Estudos de Psicologia, 26(3), 275-286. doi: 10.1590/S0103-166X2009000300001

Edwards, J. R., \& Rothbard, N. P. (2000). Mechanisms linking work and family: Clarifying the relationship between work and family constructs. The Academy of Management Review, 25(1), 178-199. doi: $10.2307 / 259269$

Fisher, G. G., Bulger, C. A., \& Smith, C. S. (2009). Beyond work and family: A measure of work/nonwork interference and enhancement. Journal of Occupational Health Psychology, 14(4), 441-456. doi: 10.1037/a0016737

Frone, M. R. (2003). Work-family balance. In J. C. Quick \& L. E., Tetrick (Orgs.), Handbook of occupational health psychology (pp.143-162). Washington: American Psychological Association.

Greenhaus, J. H., \& Beutell, N. J. (1985). Sources of conflict between work and family roles. Academy of Management Review, 10, 76-88. doi: 10.5465/AMR.1985.4277352

Greenhaus, J. H., \& Powell, G. N. (2006). When work and family are allies: A theory of work-family enrichment. Academy of Management Review, 31, 72-92. doi: 10.5465/amr.2006.19379625

Hanson, G. C., Hammer, L. B., \& Colton, C. L. (2006). Development and validation of a multidimensional scale of perceived work-family positive spillover. Journal of Occupational Health Psychology, 11(3), 249-265. doi: 10.1037/1076-8998.11.3.249
Herst, D. E. L. (2003). Cross-cultural measurement invariance of work/ family scales across english-speaking samples (Tese de doutorado não publicada). University of South Florida, Florida.

Li, C., Lu, J., \& Zhang, Y. (2013). Cross-domain effects of work-family conflict on organizational commitment and performance. Social Behavior and Personality, 41(10), 1641-1654. doi: 10.2224/ sbp.2013.41.10.1641

Lim, D. H., Morris, M. L., \& McMillan, H. S. (2011). Construct validation of the translated version of the work-family conflict scale for use in Korea. Human Resource Development Quarterly, 22(4), 519-543. doi: 10.1002/hrdq.20089

Kacmar, K. M., Crawford, W. S, Carlson, D. S., Ferguson, M., \& Whitten, D. (2014). A short and valid measure of work-family enrichment. Journal of Occupational Health Psychology, 19(1), 32-45. doi: 10.1037/a0035123

Matias, M., \& Fontaine, A. M. (2012). A conciliação de papéis profissionais e familiares: o mecanismo psicológico de spillover.Psicologia: Teoria e Pesquisa, 28(2), 235-243. doi: 10.1590/S0102-37722012000200012

Matthews, R. A., Kath, L. M., \& Barnes-Farrel, J. L. (2010). A short, valid, predictive measure of work-family conflict: Item selection and scale validation. Journal of Occupational Health Psychology, 15(1), 75-90. doi: $10.1037 / \mathrm{a} 0017443$

McMillan, H. S., Morris, M. L., \& Atchley, E. K. (2011). Constructs of the work/life interface: A synthesis of the literature and introduction of the concept of work/life harmony. Human Resource Development Review, 10(1), 6-25. doi: 10.1177/1534484310384958

McNall, L. A., Masuda, A. D., \& Nicklin, J. M. (2010). Flexible work arrangements and job satisfaction/turnover intentions: The mediating role of work-to-family enrichment. The Journal of Psychology: Interdisciplinary \& Applied, 144, 61-81. doi: 10.1080/00223980903356073

Mesmer-Magnus, J. R., \& Viswesvaran, C. (2005). Convergence between measures of work-to-family and family-to-work conflict: A meta-analytic examination. Journal of Vocational Behavior, 67(2), 215-232. doi: 10.1016/j.jvb.2004.05.004

Netemeyer, R. G., Boles, J. S., \& McMurrian, R. (1996). Development and validation of work-family conflict and family-work conflict scales. Journal of Applied Psychology, 81(4), 400-410. doi: 10.1037/0021-9010.81.4.400

Steenbergen, E. F., Ellemers, N., \& Mooijaart, A. (2007). How work and family can facilitate each other: Distinct types of work-family facilitation and outcomes for women and men. Journal of Occupational Health Psychology, 12(3), 279-300. doi: 10.1037/1076-8998.12.3.279

Sulea, C., Virgã, D., \& Galben, N. (2010). Work-family conflict scale: The analysis of the psychometric properties analysis of the romanian version. Journal of the Human Resources Psychology, 8(2), 10-20. Recuperado de http://pru.apio.ro/index.php/prujournal/article/ view/300

Wayne, J. H., Grzywacz, J. G., Carlson. D. S., \& Kacmar, K. M. (2007). Work-family facilitation: A theoretical explanation and model of primary antecedents and consequences. Human Resource Management Review, 17, 63-76. doi: 10.1016/j.hrmr.2007.01.002 
Carolina Villa Nova Aguiar, Doutora em Psicologia com concentração em Psicologia Organizacional e do Trabalho pela Universidade Federal da Bahia (UFBA), é Professora Adjunta da

Escola Bahiana de Medicina e Saúde Pública (EBMSP).

Endereço para correspondência: Rua Waldemar Falcão, 2106, apt. 2402. CEP: 40286-050. Telefone:(71) 991128839.

E-mail: carol.vna@gmail.com

Antonio Virgilio Bittencourt Bastos, Doutor em Psicologia com concentração em Psicologia Organizacional e do Trabalho pela

Universidade Federal de Brasília (UnB), é Professor Titular no Instituto de Psicologia da Universidade Federal da Bahia (UFBA).

E-mail: antoniovirgiliobastos@gmail.com

Recebido em 21.Out.16

Revisado em 06.Jul.18

Aceito em 17.Out.18 